\title{
Delay in Diagnosis of Optic Nerve and Chiasmal Compression Presenting with Unilateral Failing Vision
}

\author{
J. GARFIELD， G. NEIL-DWYER
}

British Medical fournal, 1975, 1, 22-25

\section{Summary}

Out of 29 patients who presented with failing vision in one eye due to optic nerve or chiasmal compression, compression was initially diagnosed in only five. The errors in diagnosis and lack of ophthalmological follow-up led to delays of up to many years with serious deterioration in acuity before referral for intracranial investigation. The chief causes of error were lack of charting of the visual fields, too ready acceptance of the diagnosis of neuritis in the absence of essential features, and the infrequent use of skull radiographs.

\section{Introduction}

General practitioners and, particularly, ophthalmologists face a difficult task in dealing rapidly with large numbers of patients who have a multitude of visual symptoms-whereas the neurologist and neurosurgeon have to deal only with a highly selected small group of patients with relatively rare conditions. Nevertheless, among this group are those whose failing vision is due to a remediable cause, and in these circumstances correct early diagnosis before visual acuity has declined severely can lead to treatment which can restore normal sight.

In the early diagnosis of optic nerve or chiasmal compression clinical errors continue to be made, often with disastrous results

\footnotetext{
Wessex Neurological Centre, Southampton General Hospital, Southampton SO9 4XY

J. GARFIELD, M.R.C.P., F.R.c.s., Consultant Neurosurgeon

G. NEIL-DWYER, M.B., F.R.C.S., Senior Registrar in Neurosurgery (Present address: Department of Neurosurgery, Cornwall Hospital, Montego Bay, Jamaica)
}

for preservation of vision and even prolongation of life. The initial diagnosis and decision about management are the responsibility of general practitioners, general physicians, and ophthalmologists. Papers in the specialist literature emphasizing the importance of early diagnosis are unlikely to be read by those responsible for these critical early decisions, and, though Jane and McKissock stressed in the British Medical fournal in $1962^{1}$ the importance of failing vision in the early diagnosis of suprasellar meningioma, there have been very few similar publications in the general literature since then.

The present report was prompted by the observation that in almost all patients who proved ultimately to have optic nerve or chiasmal compression and whose presenting symptoms were failing vision in one eye either the clinical diagnosis was wrong or an initial diagnosis was not made and the patient was not followed up. These mistakes had serious consequences and, surprisingly, were made as often by ophthalmologists as by general practitioners. Our purpose here is to record these errors in initial diagnosis, examine their reasons, emphasize (as others have done) the serious consequences, and, finally, suggest ways in which such errors may be avoided.

\section{Patients and Methods}

From March 1966 to February 1973, 29 patients whose chief presenting symptom was failing vision in one eye were found to have optic nerve or chiasmal compressing lesions. In 26 patients the lesion was verified at craniotomy. For this study all patients who survived and in whom the original records were not adequate were reinterviewed to obtain as full details as possible of the initial presenting symptoms and, particularly, of any diagnoses given by optician, general practitioner, or ophthalmologist. When possible previous ophthalmological notes were obtained. The decision not to operate on three patients was taken because of their age and relatively slight visual disability. Follow-up on all patients was complete. 
Details of Dalays and Diagnoses in 29 Patients with Optic Nerve or Chiasmal Compression

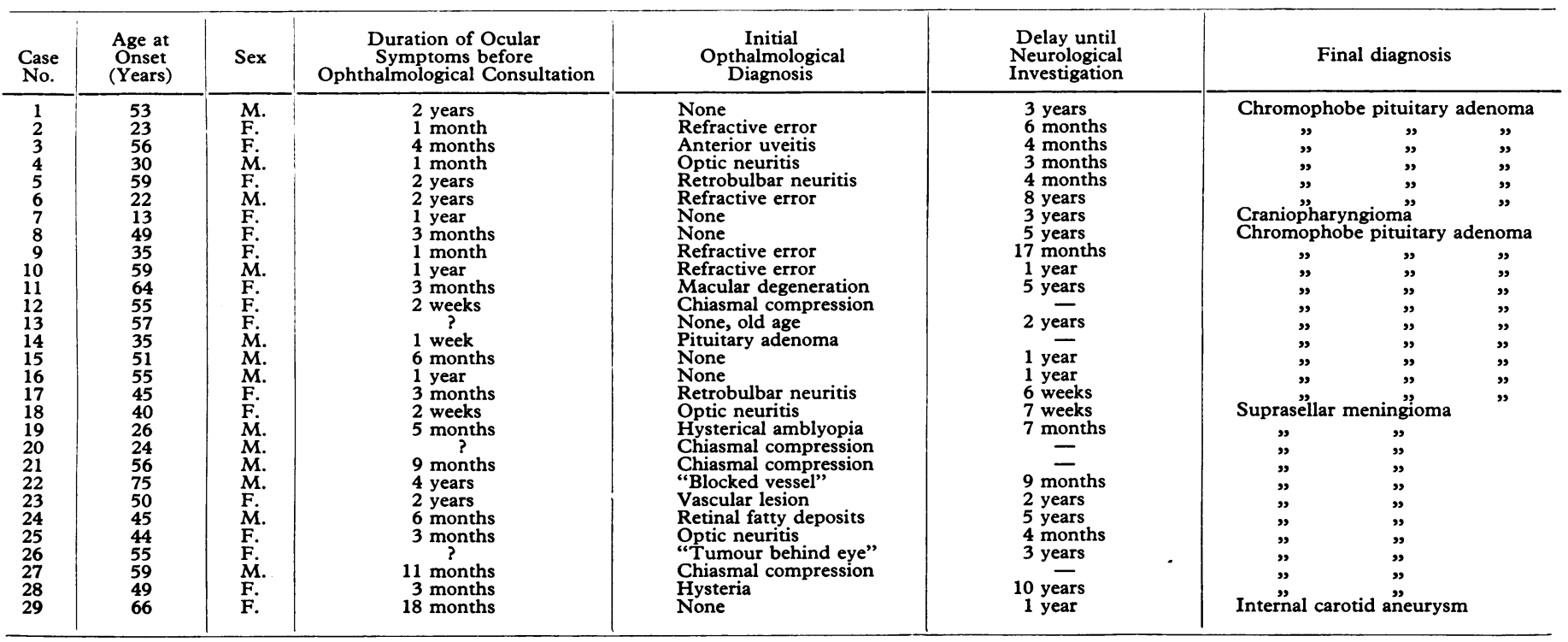

Particular attention was paid to the intervals between the onset of visual symptoms, the first ophthalmological consultation, and final referral for intracranial investigations.

\section{Results}

In only five patients was an early ophthalmological diagnosis of chiasmal or optic nerve compression made, and all these patients were referred for intracranial investigation without delay (see table). The times from onset of visual symptoms until ophthalmological diagnosis in these five, however, were nine months, 11 months, less than two weeks in two, and unknown in one.

Of the 24 patients in whom a compressing lesion was not diagnosed initially four were considered to have errors of refraction, five optic or retrobulbar neuritis, and in seven an initial diagnosis was not made. Miscellaneous diagnoses were made in the remaining eight patients, including two of vascular lesions and two of hysteria (see table).

\section{REFRACTIVE ERRORS}

The ophthalmological diagnosis of refractive errors was made initially in four patients. The presenting symptoms were diffculty in reading small print, blurred vision in one eye, intermittent blurring of vision in each in turn, and failing vision in one eye followed a year later by dementia. In none of these patients was there evidence initially of optic atrophy, and fields were not charted. The initial diagnosis led to delays of six months to eight years before referral for intracranial investigation. By then all these patients had bitemporal hemianopia, with deterioration in acuity to worse than $6 / 36$ in one eye in the three patients in whom the delay was longer than 10 months. Chromophobe pituitary adenomas were present in all four patients, but none had had skull radiographs taken before the time of referral for intracranial investigation, and by then the pituitary fossa was clearly abnormal.

\section{RETROBULBAR OR OPTIC NEURITIS}

Of the five patients in whom the initial ophthalmological diagnosis was retrobulbar or optic neuritis two had sudden deterioration in acuity to blindness or perception of light over the course of a few days. In the other three deterioration progressed steadily over four months. In only one patient was there objective evidence of a field defect initially, and that was a central scotoma, but three others later had bitemporal hemianopia. Two patients had pain in or around the affected eye at some stage, but in none was a phase of optic disc swelling observed. At referral for intracranial investigation skull radiographs showed abnormalities in four of the five patients, with an enlarged pituitary fossa in three and an enlarged optic foramen in one. None of these patients had had skull radiographs taken when the diagnosis of neuritis was made.

\section{NO OPHTHALMOLOGICAL DIAGNOSIS}

In seven patients, all of whom complained of progressive deterioration of acuity in one eye at the first ophthalmological examination, a diagnosis was not made, and in only two of these patients was ophthalmological follow-up instituted. In the other five eventual referral for intracranial investigation was prompted either by further deterioration in acuity (to perception of light or 6/60) or by the development of a bitemporal hemianopia up to five years later. In only one patient were visual fields charted at the first ophthalmological consultation, but the patient was not referred for intracranial investigation despite the presence of a temporal field defect. In another patient, a woman of 59, progressive loss of acuity with subjective awareness of a temporal field defect was ascribed at a second consultation two years later to "old age." Skull radiographs were taken initially in only one patient and showed nothing abnormal; five years later they showed only thinning of the lamina dura without any definite enlargement of the pituitary fossa, by which time the patient had a bitemporal hemianopia due to a chromophobe pituitary adenoma. Of the other six patients five had striking radiographic abnormalities of the pituitary fossa when eventually they underwent intracranial investigation.

\section{MISCELLANEOUS DIAGNOSES}

One of the two patients in whom the initial ophthalmological diagnosis was hysterical amblyopia was a 27-year-old soldier whose first symptom was a disturbance in one temporal field. The initial ophthalmic examination, however, showed nothing abnormal, but five months later when temporal hemianopia was noted the diagnosis of hysterical amblyopia was made. Abreaction failed to improve vision, and when the patient reached neurosurgical hands seven months later he was blind and remained so after removal of a suprasellar meningioma. A vascular lesion 
was diagnosed in two patients. In one, a man of 79 with a fouryear history of progressive deterioration to an acuity of $6 / 24$ without a detectable field defect, the diagnosis was of "a blocked vessel," but there was no record of any more definite ophthalmoscopic finding than slight pallor of the optic disc. In the other patient, a woman of 50 with a two-year history of progressive loss of acuity, the initial diagnosis was of a vascular lesion of the optic nerve, and there was no ophthalmic follow-up. Both these patients had suprasellar meningiomas.

In two patients confident diagnoses of retinal degenerative conditions were made, which caused delays of five years before referral for intracranial investigation. In one patient the diagnosis of macular degeneration was adhered to for four-and-a-half years while acuity declined to 6/60, and it was only when an additional upper temporal defect was noted in the other eye that a skull radiograph showing gross enlargement and erosion of the pituitary fossa was taken. The other patient was a man aged 45 who presented with sudden "patchy" loss of acuity in one eye. Fields were not charted and a very reputable ophthalmologist reported "fatty deposits and inflammatory flare up." It was not until five years later when he developed identical symptoms in the other eye that he was found to have a large suprasellar meningioma, and he died after its attempted removal.

\section{INCIDENCE OF ABNORMAL SKULL RADIOGRAPHS}

Skull radiographs were obtained in only five of the 29 patients at the initial ophthalmic referral, one showing an enlarged pituitary fossa and the other four being normal. In four patients the initial diagnosis of neuritis was revised when skull radiographs up to four months later clearly showed abnormalities. Ultimately, skull radiographs at the time of intracranial investigation showed a grossly abnormal pituitary fossa in 15 of the 16 patients with chromophobe pituitary adenomas but showed nothing abnormal in nine of the 11 patients with suprasellar meningiomas. Radiographic features were considered abnormal only if they could be seen on standard skull radiographs taken in non-specialist departments, without coned views of the pituitary fossa or tomography.

\section{Discussion}

Though neurologists, neurosurgeons, and ophthalmologists are well aware of the problem and importance of early diagnosis of optic nerve and chiasmal compression, ${ }^{1-4}$ the present study was prompted by the continuing delay in referral of this group of patients for intracranial investigation. The main reasons for delay were the interval between onset of visual symptoms and ophthalmological diagnosis, errors in initial ophthalmological diagnosis, and lack of ophthalmological follow-up. It seemed important, therefore, to examine the reasons for these errors to determine the extent to which they were due to bizarre forms of presentation or defects in clinical management.

Meadows ${ }^{2}$ pointed out that visual failure could be of sudden rather than gradual onset because an early peripheral field defect might be unnoticed by the patient, who only presented when the more vulnerable macular fibres became affected. In seven of his 11 patients an extensive, mainly central, scotomatous defect was present on one side with a normal visual field on the other at the time of the first ophthalmological examination. Elkington, ${ }^{5}$ in reporting the preoperative findings in 260 patients with pituitary adenomas, noted a unilateral central scotoma in only one. In 24 of our patients either an initial ophthalmological diagnosis was not made or it was wrong; three of these patients had unilateral central or paracentral scotomata, the initial diagnoses being optic neuritis, macular degeneration, and "blocked vessel." In two other patients the visual loss was sudden and was diagnosed as neuritis in both cases. Therefore, the mode of presentation was not unduly bizarre and does not explain the delays and errors in diagnosis.

\section{FIELD CHARTING}

Clover $^{3}$ noted wrong initial ophthalmological diagnoses in 30 out of 100 patients with proved pituitary tumours; in 14 the diagnosis was refractive error. Holmes-Sellors ${ }^{6}$ emphasized that a refractive error may be present in association with a compressing lesion and that correction of the refractive error with improvement in vision may obscure the true diagnosis. In the present series four patients were considered to have refractive errors and spectacles were changed at intervals, but the ophthalmic follow-up was irregular and without review of the visual fields. Diagnosis was delayed until there was severe loss of acuity, optic atrophy, an obvious bitemporal hemianopia, or an abnormal pituitary fossa when skull radiographs were eventually requested. In these patients the most serious omission was either lack of initial charting of the visual fields or lack of repeated charting despite the progressive loss of acuity. HolmesSellors ${ }^{6}$ has stated clearly that field charting should always be used when there is no obvious explanation of visual difficulties. Ehlers and Malmros, ${ }^{7}$ in a review of suprasellar meningioma, concluded that several patients were "coming to operation at a too advanced stage because the ophthalmologist did not examine the visual field at the early consultation of the patient." Similarly, Pruett and Wepsic ${ }^{4}$ found that the late diagnosis of chiasmal compression by ophthalmologists in nine patients was because of failure to chart visual fields, misinterpretation of symptoms and visual field defects, failure to obtain skull radiographs, and inadequate follow-up.

The failure to arrive at a definite diagnosis in seven of our patients is disturbing, particularly as formal charting of the visual fields was done in only one. In six patients either the loss of acuity progressed relentlessly to perception of light or $6 / 60$ or they were later found to have hemianopic defects. Furthermore, ophthalmological follow-up was instituted in only one of the patients in whom a diagnosis was not made, and this failure undoubtedly contributed to the serious deterioration in acuity before a remedy was sought. It seems that general practitioners were not sufficiently aware that the absence of a definite ophthalmological diagnosis did not exclude the presence of a compressing lesion, obvious as it may seem in retrospect.

\section{CRITERIA FOR DIAGNOSING NEURITIS}

Diagnosing optic or retrobulbar neuritis instead of compression is a well-known pitfall. Meadows ${ }^{2}$ and Holmes-Sellors ${ }^{6}$ have emphasized the importance of suspecting a compressing lesion when acuity does not improve, and Holmes-Sellors stated that retrobulbar neuritis should be diagnosed only when declining vision is accompanied by pain and altered pupillary reflexes. Of the five patients in whom neuritis was diagnosed only one had had a true attack of pain in the affected eye at the onset of visual loss, and one other had had pain in and around the eye for seven months. Fortunately, ophthalmological follow-up was considerably more thorough than in the patients without any definite diagnosis but, nevertheless, there were delays of up to four months before neurological referrral. Had the citeria for the diagnosis of neuritis been more strictly observed and plain skull radiographs taken the correct diagnosis in four of the patients would have been made much earlier.

Jane and $M c K i s s o c k{ }^{1}$ in reviewing 33 patients with suprasellar meningiomas noted radiographic skull abnormalities in only 16 , and Ehlers and Malmros ${ }^{7}$ found abnormalities preoperatively in only four of their 31 patients though this number rose when the radiographs were reviewed in the light of the operative findings. This low incidence of radiographic abnormalities in suprasellar meningiomas has also been emphasized by others, including Meadows. ${ }^{2}$ Since 16 of our patients had chromophobe pituitary 
adenomas (which commonly expand the pituitary fossa before or soon after the onset of visual symptoms), however, the use of skull radiographs earlier would probably have hastened the correct diagnosis in most patients.

\section{Conclusions}

The main reason for errors in the diagnosis of optic nerve or chiasmal compression in patients presenting with unilateral failing vision is failure to think of the condition. Omitting to chart the visual fields, lack of regular ophthalmological follow-up in patients in whom an unequivocal diagnosis could not be made initially, and infrequent use of skull radiographs all contributed to this failure.

Despite the rarity of compressing lesions and the variation in their mode of presentation we believe that most errors in diagnosis could be avoided if the following points were always considered: ( $a$ ) the diagnosis of a compressing lesion should be entertained whenever unilateral failing vision does not have an unequivocally demonstrable intraocular cause; $(b)$ a diagnosis of retrobulbar or optic neuritis should not be accepted without the essential criteria followed by the usual course of this con- dition; (c) the visual fields should be charted early and repeatedly; $(d)$ patients in whom a firm diagnosis cannot be made at the first consultation should be carefully followed up; (e) plain skull radiographs should be requested in those patients in whom a firm diagnosis cannot be made and in those in whom a compressing lesion is suspected.

We thank our neurological and neurosurgical colleagues of the Wessex Neurological Centre for permission to repont patients admitted under their care and Dr. Peter Robinson for advice in the preparation of this paper.

\section{References}

1 Jane, J. A., and McKissock, W., British Medical fournal, 1962, 2, 5. Meadows, S. P., Proceedings of the Royal Society of Medicine, 1949, 42 1017.

3 Clover, P., Proceedings of the Royal Society of Medicine, 1961, 54, 616. 4 Pruett, R. C., and Wepsic, J. G., American Fournal of Ophthalmology, $1973,76,229$

Elkington, S. G., British fournal of Ophthalmology, 1968, 52, 322.

${ }^{6}$ Holmes-Sellors, P., in Pituitary Tumours, ed. J. S. Jenkins, p. 64. London, Butterworths, 1973.

7 Ehlers, N., and Malmros, R., Acta Ophthalmologica, 1973, Suppl. 121.

\title{
Hospital Topics
}

\section{Thyroid Function after Subtotal Thyroidectomy for Hyperthyroidism}

\author{
DAVID EVERED, E. T. YOUNG, W. M. G. TUNBRIDGE, B. J. ORMSTON, E. GREEN, \\ V. B. PETERSEN, P. H. DICKINSON
}

Brtish Medical fournal, 1975, 1, 25-27

\section{Summary}

Among 76 patients who had had a subtotal thyroidectomy for hyperthyroidism from one to seven years previously recurrent hyperthyroidism was found in three and hypothyroidism in 13. The remaining 60 subjects were clinically euthyroid but a raised level of serum thyroid-stimulating hormone (TSH; $>5.0 \mu \mathrm{U} / \mathrm{ml}$ ) was found in 39. Analysis of the data showed that their serum thyroxine was significantly lower than in the subjects with a normal TSH. The serum triiodothyronine ( $T-3)$ was similar in both groups. It is concluded that subjects with a raised TSH remain clinically euthyroid by maintaining a normal serum T-3 concentration. There was no evidence of any long-term progressive deterioration of thyroid function after subtotal thyroidectomy.

\footnotetext{
Department of Medicine, University of Newcastle upon Tyne and Royal Victoria Infirmary, Newcastle upon Tyne NE1 4LP

DAVID EVERED, M.D., M.R.C.P., Consultant Physician

E. T. YOUNG, M.B., M.R.C.P., Consultant Physician

W. M. G. TUNBRIDGE, M.B., M.R.C.P., Senior Research Associate

B. J. ORMSTON, M.B., M.R.C.P., Research Registrar

E. GREEN, Laboratory Technician

V. B. PETERSEN, M.SC., Biochemist

P. H. DICKINSON, M.S., F.R.C.S., Consultant Surgeon
}

Introduction

Subtotal thyroidectomy is commonly practised in the management of hyperthyroidism. The incidence of hypothyroidism may lie between $3 \%$ and $35 \%$ and that of recurrent hyperthyroidism between $1.2 \%$ and $28 \% .^{1}$ Hedley et al. ${ }^{2-4}$ have found a recurrence rate in the north-east of Scotland of $6 \%$ for hyperthyroidism and a point prevalence for hypothyroidism of $35 \%$, but they also found a raised serum thyroid-stimulating hormone (TSH; $>5 \mu \mathrm{U} / \mathrm{ml})$ in $40 \%$ of their subjects who were unequivocally euthyroid clinically and on the basis of routine biochemical investigations. The present study was undertaken to establish the level of thyroid function after subtotal thyroidectomy in a group of subjects from north-east England and to assess the significance of a raised serum TSH by measuring serum thyroxine $(T-4)$ and triiodothyronine $(T-3)$ concentrations.

\section{Patients and Methods}

All patients under the care of one surgeon (P.H.D.) who had undergone subtotal thyroidectomy for thyrotoxicosis during 1966, 1969, and 1972 were selected for study. All the operations were carried out by the surgeon, whose technique did not vary during this period. Eighty-nine subjects were selected for study, of whom eight had left the area and could not be traced and five had died from unrelated causes. Nine of the remaining 76 patients had been started on thyroxine after 